\title{
Ares I Static Tests Design
}

\author{
William C. Carson, Kathleen M. Lindemuth, John P. Mich, K. Preston White, Jr., Ph.D., Senior \\ Member, IEEE, and Peter A. Parker, Ph.D.
}

\begin{abstract}
Probabilistic engineering design enhances safety and reduces costs by incorporating risk assessment directly into the design process. In this paper, we assess the format of the quantitative metrics for the vehicle which will replace the Space Shuttle, the Ares I rocket. Specifically, we address the metrics for in-flight measurement error in the vector position of the motor nozzle, dictated by limits on guidance, navigation, and control systems. Analyses include the propagation of error from measured to derived parameters, the time-series of dwell points for the duty cycle during static tests, and commanded versus achieved yaw angle during tests. Based on these analyses, we recommend a probabilistic template for specifying the maximum error in angular displacement and radial offset for the nozzle-position vector. Criteria for evaluating individual tests and risky decisions also are developed.
\end{abstract}

\section{INTRODUCTION}

$\mathrm{T}$ HE National Aeronautics and Space Administration (NASA) plans to retire the Space Shuttle in 2010. Under NASA's Constellation Program, the Orion spacecraft and Ares series of rockets are set to replace the shuttle as the principal method for transporting astronauts and cargo into space. NASA's Langley Research Center in Hampton, Virginia, is responsible for developing the static testing experiments required to evaluate the capabilities of Ares I rocket motors. The University of Virginia was tasked with specifying Ares I performance for: 1) thrust-vector angular misalignment; and, 2) thrust-vector radial offset during firing. Limits are required on each of these variables in order to comply with the limits on guidance, navigation, and control systems during flight.

Specification is based on principles of probabilistic engineering design applied to statistical analysis of the Ares I static tests in order to verify performance. Traditionally, engineers design to input variables which are deterministic they are set at either worst-case or nominal values. Calculating reliability based on worst-case values results in

Manuscript received April 6, 2009. This work was supported by the National Institute of Aerospace (NASA \#NNL08AA00B, NIA Task Order $6238)$.

William C. Carson, Kathleen M. Lindemuth, and John P. Mich are undergraduate students in the University of Virginia School of Engineering and Applied Science's Department of Systems Engineering, 151 Engineer's Way, Charlottesville, VA 22904 USA (e-mail: wcc9r@virginia.edu, km14t@virginia.edu, and jpm2j@virginia.edu, respectively).

K. Preston White, Jr., Ph.D., is a professor in the University of Virginia School of Engineering and Applied Science's Department of Systems Engineering, 151 Engineer's Way, Charlottesville, VA 22904 USA (phone: 434-982-2070; e-mail: kpw8h@ virginia.edu).

Peter A. Parker is with the National Aeronautics and Space Administration's Langley Research Center, Hampton, VA 23681 USA (email: Peter.A.Parker@nasa.gov). over-design and unnecessarily large development and production costs. Alternately, adopting nominal scenarios requires that a factor of safety be applied to the resulting nominal design. Prudent safety factors also result in overdesign and attendant costs. Without a rigorous treatment of the uncertainties in the input variables, there is no way to measure the potential over-design penalty in either case.

By contrast, probabilistic engineering design addresses uncertainties explicitly [1], [2], [3]. Probability distributions are estimated for uncertain input variables. These estimates are propagated through the analysis, resulting in distributions for the output variables. Because this approach directly influences reliability, instead of designing to the worst case or safety-adjusted nominal, a probabilistic risk assessment is built into the design process. The probabilistic methodology provides a greatly improved understanding of reliability bounds which, in turn, can both enhance safety and reduce costs.

The work reported here focuses on the analysis of previous static tests, in order to determine how these and future tests interface with probabilistic performance metrics of the Ares I design. Since test data are costly and scarce, sophisticated inference is required to determine whether performance has been met under acceptable levels of risk. Materials describing the static tests were reviewed and errors were detected in derivation of the static model [4]. It was determined that these errors were not incorporated into the static model and that the model appears to be sound under the reported assumptions. This report develops propagationof-error, dwell-point, and input-output analyses, continues with a template for writing unambiguous and testable probabilistic metrics, and closes with a discussion of decisions and risk equilibrium.

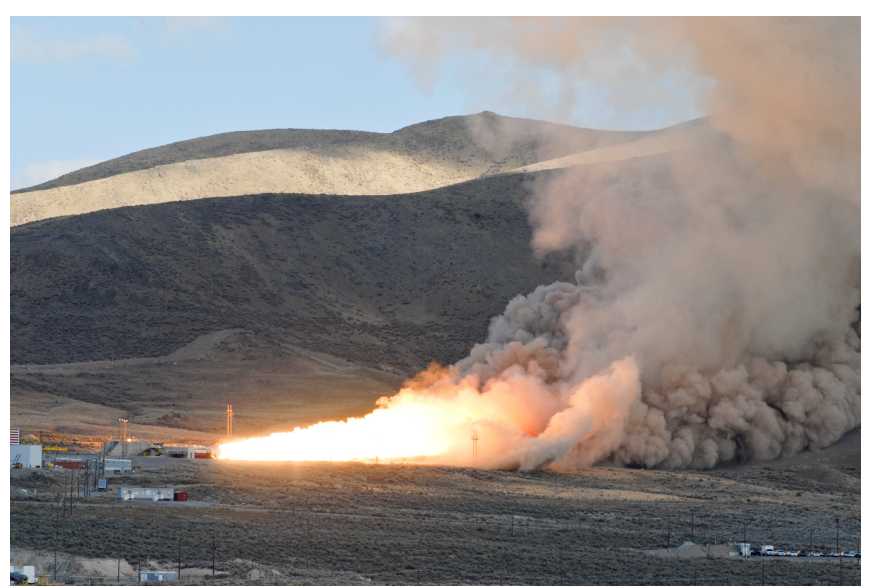

Fig. 1. Fire and smoke billow from a space shuttle reusable solid rocket motor static test at a Utah test facility. [5] 


\section{PROPAGATION-OF-ERROR ANALYSIS}

With respect to the functional relationship between the derived parameter and its measured constituents, derived parameter uncertainty is the aggregate of the error in each measured parameter. Since functional relationships are often based on derived parameters, propagated error directly influences whether a design will be accepted or rejected, correctly or incorrectly.

Consider a motor nozzle vectored during static testing. There are two variables which determine angular displacement, ANGDISP (see Fig. 2): THETAY and SDELY. THETAY, the dynamic thrust vector, is the angle between the test-bay centerline and the motor thrust vector determined from longitudinal and lateral test stand thrust measurements ( $F_{X}$ and $F_{Y}$, respectively), given by:

$$
\text { THETAY }=\arctan \left(\frac{F_{Y}}{F_{X}}\right) .
$$

SDELY, the nozzle vector angle, is the angle between the test-bay vector and the nozzle centerline calculated using nozzle extensometer data [6]. ANGDISP is defined as the difference between these two parameters:

\section{$A N G D I S P=T H E T A Y-S D E L Y$.}

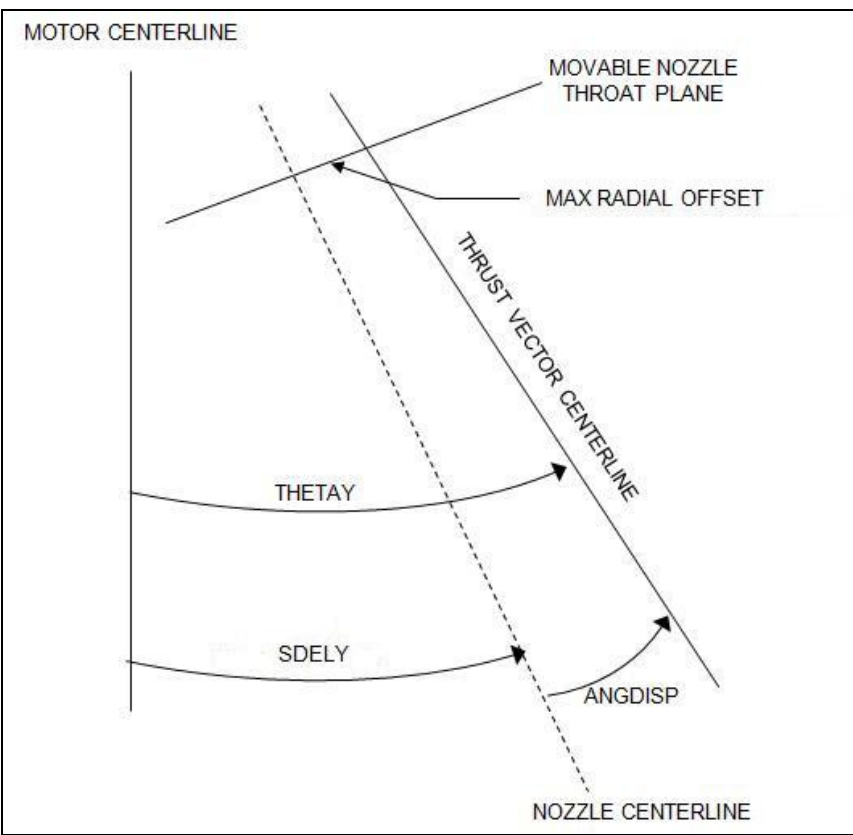

Fig. 2. Line diagram depicting parameters of interest (ANGDISP, THETAY, and $S D E L Y$ ) for the propagation-of-error analysis. [6]

Deterministic target performance for $A N G D I S P$ is given by:

$$
|A N G D I S P| \leq\left\{\begin{array}{ll}
X^{\circ}, & t_{1} \leq t<t_{2} \\
Y^{\circ}, & t \geq t_{2}
\end{array},\right.
$$

where $t$ is time measured from the beginning of the test firing. We wanted to know the proportion of this ANGDISP metric potentially consumed by measurement errors. In the Appendix, we determined the functional form for the error in $A N G D I S P$, as propagated through errors in THETAY and SDELY:

$$
\sigma_{A N G D I S P} \leq \frac{\left(\frac{F_{Y}}{F_{X}}\right) \sqrt{\left(\frac{\sigma_{F_{Y}}}{F_{Y}}\right)^{2}+\left(\frac{\sigma_{F_{X}}}{F_{X}}\right)^{2}}}{1+\left(F_{Y} / F_{X}\right)^{2}}+\sigma_{S D E L Y}
$$

From this inequality, we see that (ceteris paribus) as longitudinal thrust, $F_{X}$, increases, the error in ANGDISP is driven to, at most, the error in SDELY (since the uncertainty in SDELY is unknown). This result is likely in static testing scenarios as the magnitude of longitudinal thrust is much greater than that of lateral thrust for $t \geq t_{l}$. To determine the maximum error in ANGDISP under unlikely situations, we evaluated the inequality with maximum and minimum values for yaw and thrust, respectively, from previous static tests for $t \geq t_{1}$. Thus, we set yaw to the maximum yaw angle from static test 14 and thrust to nominal minus 25 percent; $\sigma_{F Y}$ and $\sigma_{F X}$ are set to known values. Thus, the error of ANGDISP is given by:

$$
\sigma_{A N G D I S P} \leq X X X+\sigma_{S D E L Y}
$$

where $X X X$ is small relative to the uncertainty in SDELY.

Radial offset at the throat plane (TRO) is defined as the offset distance between the nozzle centerline and the dynamic thrust vector centerline determined at the throat plane and is approximated by:

$$
T R O \approx \frac{-F S F \times L_{Y}}{F_{X}}+Y_{00},
$$

where FSF is forward side force, $L_{Y}$ is distance between the forward and aft test stands, and $Y_{O 0}$ is nozzle centerline offset in the $y$-axis (as determined from the extensometers - this parameter is a function of SDELY). Deterministic target performance for $T R O$ is given by:

$$
|T R O| \leq X \text { in },
$$

for all $t$. In the Appendix, we determined the functional form for the error in $T R O$, as propagated through the errors across its constituent parameters:

$$
\begin{aligned}
& \sigma_{T R O} \leq \sigma_{Y_{00}}+\left(\frac{-F S F \times L_{Y}}{F_{X}}\right) \times \\
& \sqrt{\left(\frac{\sigma_{F S F \times L_{Y}}}{F S F \times L_{Y}}\right)^{2}+\left(\frac{\sigma_{F_{X}}}{F_{X}}\right)^{2}+2\left(\frac{\sigma_{F S F \times L_{Y}}}{F S F \times L_{Y}}\right)\left(\frac{\sigma_{F_{X}}}{F_{X}}\right) \rho_{F S F \times L_{Y}, F_{X}}}
\end{aligned}
$$

Once again, we see that (ceteris paribus) as longitudinal 
thrust, $F_{X}$, increases, the error in TRO is driven to, at most, the error in $Y_{O O}$ (since the uncertainty in $Y_{O O}$ is unknown). Thus, uncertainties in $A N G D I S P$ and $T R O$ are almost entirely dependent on uncertainties in SDELY or a function thereof $\left(Y_{00}\right)$, even in an extreme-case yaw/thrust scenario. Thus, if the uncertainty in SDELY is extremely large, improvements to the extensometers which improve their precision may be a viable option to prevent rejection of a suitable design. Otherwise, improvements to the nozzle positioning hardware may be more crucial to ensuring that $A N G D I S P$ and TRO are within specification during the static tests.

\section{DWELL-POINT ANALYSIS}

The duty cycle for a static test is designed to characterize a motor's performance with limited resources. The duty cycle serves as a representation for various motor movements within flight (see Fig. 3). Within a given static test, the yaw angle oscillates between $X \pm Y$ degrees for $t=t_{1}$ to $t_{2}$. Throughout this period, there are seven similar dwell points which occur at each crest and trough of the oscillatory wave. Since the vectored motor is never completely motionless, a dwell point is thus defined as a small time interval within the oscillatory period during which yaw angle does not deviate more than $X$ degrees. The primary purposes of this dwell point analysis are to: 1) compare the within-test and across-test variation; and, 2) determine if the presence of an oscillatory pattern within a duty cycle provides information that cannot be gathered by other designs.

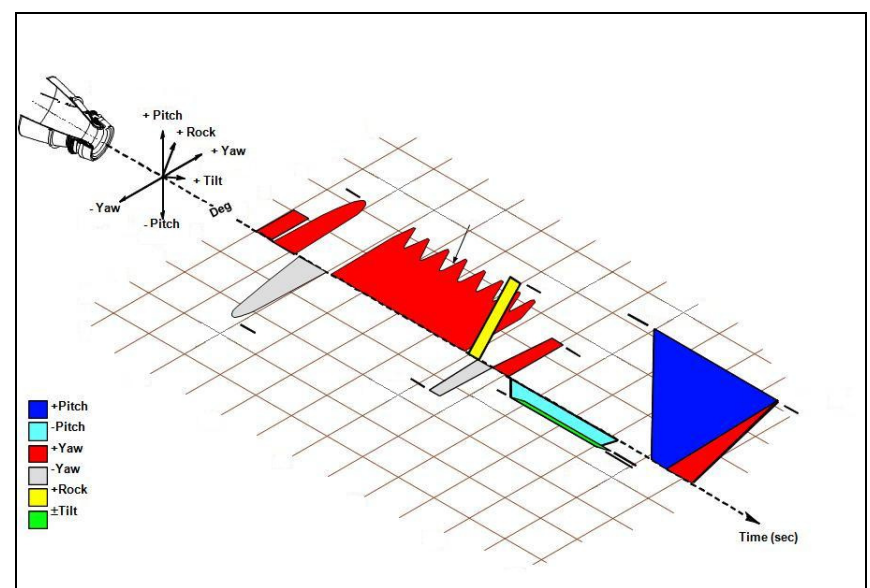

Fig. 3. Current reusable solid rocket motor duty cycle for Ares I static testing. Dwell points occur in sawtooth pattern towards middle of graph.

First, we performed an ANOVA to compare within-test ANGDISP values at each of the dwell points. The ANOVA showed that the ANGDISP values were statistically different across dwell points within each test (see Fig. 4). Further box plot analysis revealed that ANGDISP values were significantly different across runs at like dwell points (see Fig. 5). Yaw angles and ANGDISP values suggest that static test 11 was most unlike the other three tests. After the data from this test were removed from the set, additional ANOVA and box plots were generated. The data from the remaining runs show that ANGDISP still differs significantly across tests, even with the removal of test 11 .

In order to characterize ANGDISP values within and across runs, we plotted the yaw angles of dwell points from static tests 11-14 and discovered that: 1) the yaw angles at dwell points appear to increase within runs; and, 2) for a given dwell point, yaw angles are different across runs.

The differences in yaw angles across tests are statistically significant, but their practical significance is suspect. Prior to this work, NASA knew that yaw angle is not constant within and across tests, and understood that nominal yaw angle at the dwell points according to the duty cycle is a nominal target. Additionally, the parabolic trend seen for yaw angle within tests was classified by NASA as a known phenomenon.

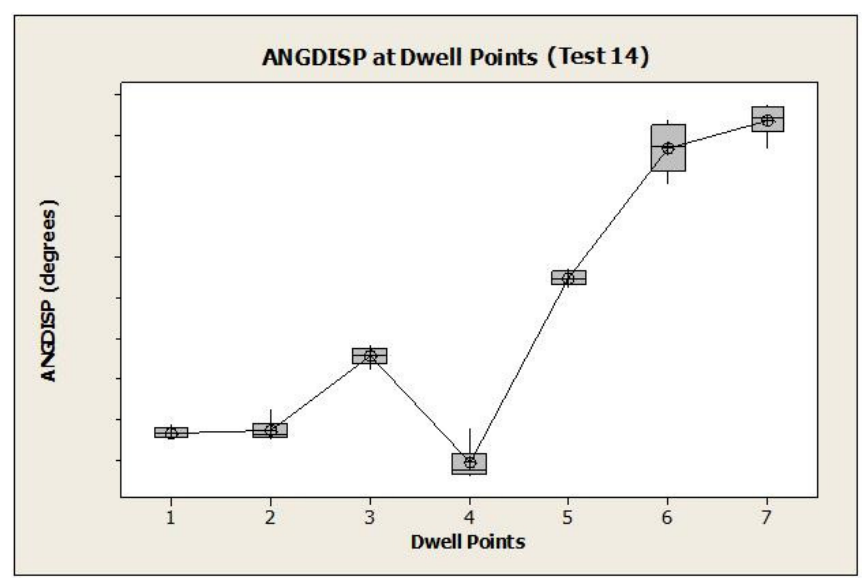

Fig. 4. Box plot of ANGDISP at dwell points for static test 14. These data exhibit within-test variation (an increase in ANGDISP over $t$ ).

Both the crest and trough analyses show that yaw angles in static test 11 are significantly lower than in the other three tests. Additionally, ANGDISP values are not equal to one another across runs at a statistically significant level, with those in static test 11 being higher than in the other three tests. The correlation between ANGDISP and yaw angle is explored in the following section.

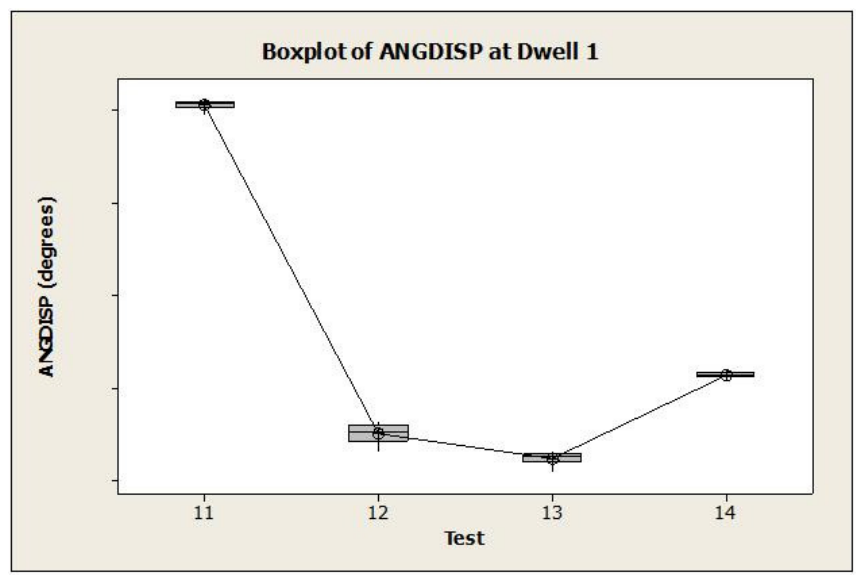

Fig. 5. Box plot of ANGDISP at dwell point 1 across tests. ANGDISP in test 11 are statistically (but may not be practically) significantly different than ANGDISP in the other tests. 
From this analysis, we conclude that: 1) yaw angles at dwell points increase across time within tests and differ across tests, a phenomenon observed by NASA; 2) $A N G D I S P$ is not correlated with yaw angle (at this point in the analysis); and, 3) within each test, yaw angle increases along $t$ for oscillatory dwell point data. Furthermore, at Dwell 1, static tests 12-14 have ANGDISP well within the error range calculated in the first section. Moreover, ANGDISP in static test 11 is within the target unless the uncertainty from the error propagation formulae is exceedingly large (more than three times the estimated value). The difference among the tests suggests that there is across-test (rocket-to-rocket) variation in these data, which could be caused by the control systems, rocket hardware, fuel mixture, or a host of other factors.

\section{INPUT-OUTPUT ANALYSIS}

Each trial's yaw pitch angle distributions have the same approximate multimodal shapes, but there are discrepancies in the frequencies of commanded angles (consider dwellpoint analysis in Section III). These discrepancies affect the theoretical output distributions of ANGDISP and TRO, which make across-trial analysis and inference difficult. That is, both test-to-test variation from exogenous variables and variation from command variables are present in TRO and ANGDISP.

In Fig. 6, the four scatterplots of angular misalignment versus yaw exhibit positive slopes. That is, as yaw angle increases, angular misalignment increases, as well. Simple linear regression models were fit to these data to confirm this observation. In each trial, the slope of the regression model was found to be significant and positive.

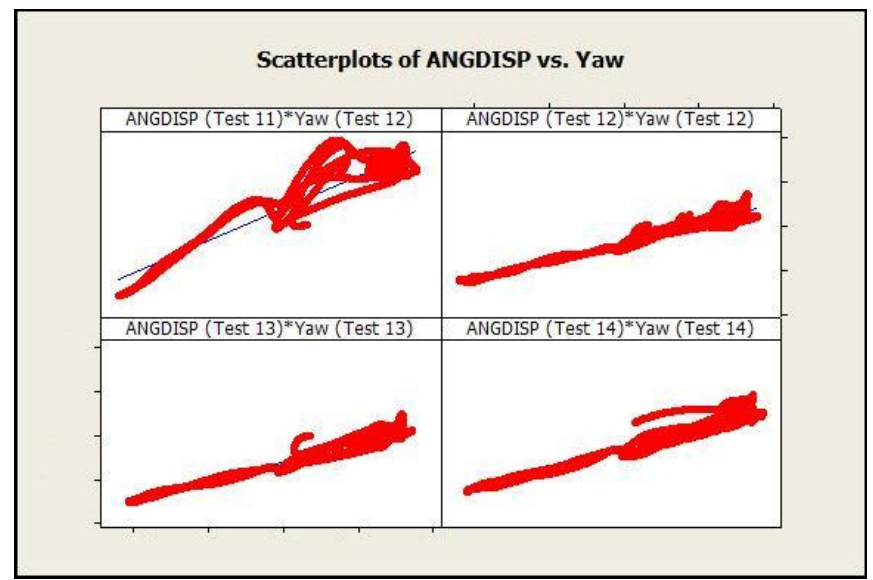

Fig. 6. Scatterplots of ANGDISP vs. Yaw. Notice how the slope of the ANGDISP vs. Yaw relationship for static test 11is steeper than those of static tests 12 through 14 .

In Fig. 7, the four scatterplots of radial offset vs. yaw appear to exhibit a negative slope. That is, as yaw angle increases, radial offset decreases. Simple linear regression models were fit to these data to confirm this observation. In each trial, the slope of the regression model was found to be significant and negative. As yaw increases, variance in angular misalignment and radial offset increase, as well.

In order to identify when during each firing this occurs, the residuals from the linear regression models were plotted against time. This revealed that large residuals come towards the end of the firing cycle for both ANGDISP and $T R O$, and also at the beginning of the firing cycle for TRO. Since pitch is radically manipulated at the end of the duty cycle, ANGDISP and TRO were each plotted against pitch for the four test firings. The most extreme variance occurs when pitch is approximately zero, which suggests another factor influences $A N G D I S P$ and $T R O$ variability at the end of the duty cycle, which we hypothesize is change in thrust. This is confirmed by the contour plot in Fig. 8.

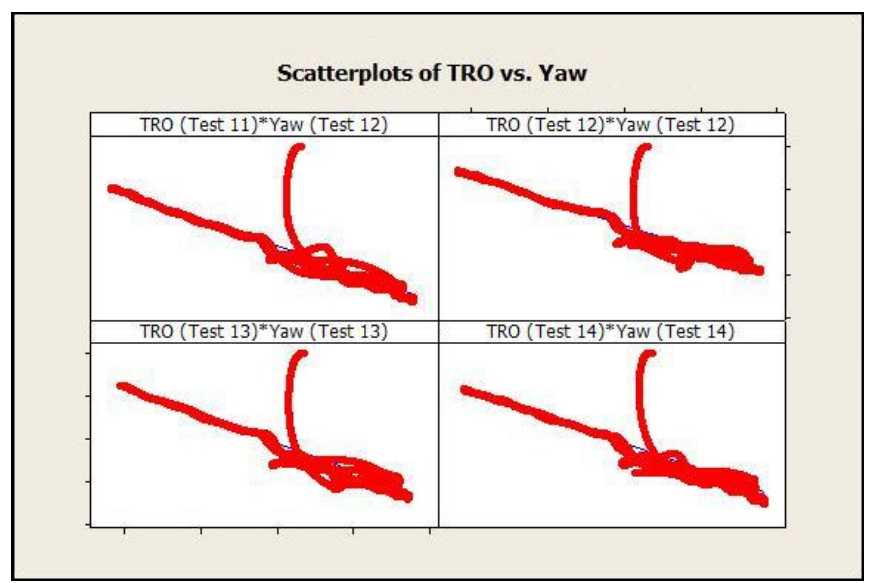

Fig. 7. Scatterplots of TRO vs. Yaw.

The contour plot in Fig. 8 also brings to attention a bias in $A N G D I S P$ caused by motor nozzle orientation, as there is no symmetry about the $Y a w=c$ axis.

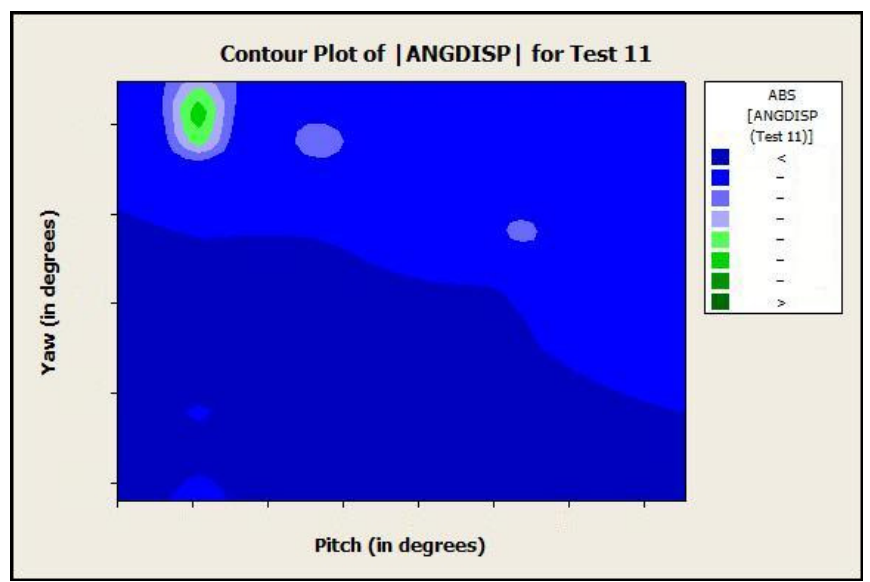

Fig. 8. Contour plot of the absolute value of ANGDISP vs. Yaw vs. Pitch for test 11. IANGDISPI is greatest when Yaw is large and pitch is small. This suggests ANGDISP is affected by the sawtooth pattern in the duty cycle.

\section{Writing Probabilistic Metrics}

Typical performance metrics for ANGDISP and TRO could be written as follows [6]:

ANGDISP: Misalignment of the dynamic thrust vector with 
respect to the nozzle centerline, shall deviate less than or equal to $\pm X . X X$ degrees for $T=t_{1}$ to $T=t_{2}$ and $\pm Y$.YY degrees for $T>t_{2} \ldots$

TRO: The radial offset between the dynamic thrust vector and nozzle centerline at the movable nozzle throat plane shall be less than or equal to X.XX inches...

These metrics could be refined from a deterministic paradigm to address the probabilistic nature of acceptance sampling. As these are written, any datum recorded in a test firing which exceeds the specifications causes the run to be a failure. We refined the metrics to address the probabilities of both Type I and Type II error. Reliability, $\rho$, is the minimum proportion of data within specification and $\varphi$ is a parameter of interest. The design goal is to obtain a proportion of $\varphi$ above or below some threshold, $\varphi_{M I N}$ or $\varphi_{M A X}$, which meets or exceeds the minimum reliability, which is related to Type I error:

$$
\operatorname{Pr}\left[\varphi \leq \varphi_{M A X}\right] \geq[\rho=1-\alpha] .
$$

Thus, design goals can be written in a probabilistic form. For example:

P\% (reliability) of all observations in a given test must be less than $Y$ (threshold value) with $Z \%$ of $\beta$ risk (determined by size of data pool; Power $=1-\beta$ ).

For $A N G D I S P$ and TRO:

ANGDISP: P\% of observations of misalignment of the dynamic thrust vector with respect to the nozzle centerline, shall deviate less than or equal to $\pm X$.XX for $T=t_{1}$ to $T=t_{2}$ and $\pm Y$. $Y Y$ degrees for $T>t_{2}$ with $Z \%$ of $\beta$ risk.

TRO: P\% of observations of radial offset between the dynamic thrust vector and nozzle centerline at the movable nozzle throat plane shall be less than or equal to $X . X X$ inches with Z\% of $\beta$ risk.

Reliability and desired power are parameters which are determined by project managers. Selection of these parameters involves careful risk and cost-benefit analyses.

\section{DECISIONS AND RISK EQUILIBRIUM}

In order to characterize the risk attitude of a missioncritical decision relating to performance, we developed the following expressions:

$$
C_{D} \alpha \text { and } C_{F} \beta \pi
$$

where $C_{D}$ is the expected cost of preventable delays from suspending operations, $\alpha$ is the probability of Type I error (the probability of incorrectly rejecting a suitable design or "producer's risk"), $C_{F}$ is the expected cost of preventable failures from continuing operations, $\beta$ is the probability of Type II error (the probability of incorrectly accepting an unsuitable design or "consumer's risk"), and $\pi$ is the expected probability of the unsuitable design causing failures of cost $C_{F}$.

The ideal scenario, in which the decision-making process is risk-neutral, occurs when:

$$
C_{D} \alpha=C_{F} \beta \pi
$$

The decision-making process is risk-averse if:

$$
C_{D} \alpha>C_{F} \beta \pi,
$$

otherwise, it is excessively risk-seeking. Risk neutrality is the preferred long-run decision-making paradigm, as the expected value of the sum of incurred losses due to preventable delays and preventable failures is minimized. Accurate estimations of the cost parameters are essential to choosing $\alpha$ and $\beta$, such that risk is neutralized. However, many costs are not explicit and do not scale with respect to magnitude. For example, what is the dollar value of an astronaut? Is it greater than that of an engineer or a civilian? Is the cost of delaying launch for 30 days the same as the cost of delaying launch for a day multiplied by 30 ? Biases in parameter estimation lead to unaccounted risk, which may lead to unintentional risk-seeking or risk-averse decisionmaking.

\section{APPENDIX}

\section{A.l Propagation of error in ANGDISP:}

$\sigma_{\text {ANGDISP }} \leq \sigma_{\text {THETAY }}+\sigma_{\text {SDELY }}$

where...

$\sigma_{\text {THETAY }}=\sigma_{\arctan (x)}$

$\sigma_{\arctan (x)}^{2}=\left(\frac{\partial \arctan (x)}{\partial x} \sigma_{x}\right)^{2}$

$\sigma_{\arctan (x)}=\frac{\partial \arctan (x)}{\partial x} \sigma_{x}=\frac{\sigma_{x}}{1+x^{2}}=\frac{\sigma_{F Y / F X}}{1+\left(F_{Y} / F_{X}\right)^{2}}$

where...

$\sigma_{F_{Y} / F_{X}}=\left(\frac{F_{Y}}{F_{X}}\right) \sqrt{\left(\frac{\sigma_{F_{Y}}}{F_{Y}}\right)^{2}+\left(\frac{\sigma_{F_{X}}}{F_{X}}\right)^{2}-2\left(\frac{\sigma_{F_{Y}}}{F_{Y}}\right)\left(\frac{\sigma_{F_{X}}}{F_{X}}\right) \rho_{F_{Y}, F_{X}}}$

and...

$F_{Y}=$ Thrust $\times \sin (90-$ Yaw $), F_{X}=$ Thrust $\times \cos (90-$ Yaw $)$

assume...

$\rho_{F_{Y}, F_{X}}=0$ because load cells are rigid

thus...

$\sigma_{A N G D I S P} \leq \frac{\left(\frac{F_{Y}}{F_{X}}\right) \sqrt{\left(\frac{\sigma_{F_{Y}}}{F_{Y}}\right)^{2}+\left(\frac{\sigma_{F_{X}}}{F_{X}}\right)^{2}}}{1+\left(F_{Y} / F_{X}\right)^{2}}+\sigma_{S D E L Y}$ 
$\sigma_{T R O} \leq \sigma_{\frac{-F S F \times L_{Y}}{F_{X}}}+\sigma_{Y_{00}}$

where...

$\frac{\sigma_{-F S F \times L_{Y}}}{F_{X}}=\left(\frac{-F S F \times L_{Y}}{F_{X}}\right) \times$

$\sqrt{\left(\frac{\sigma_{F S F \times L_{Y}}}{F S F \times L_{Y}}\right)^{2}+\left(\frac{\sigma_{F_{X}}}{F_{X}}\right)^{2}+2\left(\frac{\sigma_{F S F \times L_{Y}}}{F S F \times L_{Y}}\right)\left(\frac{\sigma_{F_{X}}}{F_{X}}\right) \rho_{F S F \times L_{Y}, F_{X}}}$

and...

$\sigma_{-F S F \times L_{Y}}=\left(-F S F \times L_{Y}\right) \times$

$\sqrt{\left(\frac{\sigma_{F S F}}{F S F}\right)^{2}+\left(\frac{\sigma_{L_{Y}}}{L_{Y}}\right)^{2}+2\left(\frac{\sigma_{F S F}}{F S F}\right)\left(\frac{\sigma_{L_{Y}}}{L_{Y}}\right) \rho_{F S F, L_{Y}}}$

so...

$\sigma_{T R O} \leq \sigma_{Y_{00}}+\left(\frac{-F S F \times L_{Y}}{F_{X}}\right) \times$

$\sqrt{\left(\frac{\sigma_{F S F \times L_{Y}}}{F S F \times L_{Y}}\right)^{2}+\left(\frac{\sigma_{F_{X}}}{F_{X}}\right)^{2}+2\left(\frac{\sigma_{F S F \times L_{Y}}}{F S F \times L_{Y}}\right)\left(\frac{\sigma_{F_{X}}}{F_{X}}\right) \rho_{F S F \times L_{Y}, F_{X}}}$

\section{REFERENCES}

[1] T. A. Craney, "Probabilistic engineering design," Reliability Review: The R\&M Engineering Journal, vol. 23-2, Jun. 2003.

[2] D. P. Giesy, L. G. Crespo, and S. P. Kenny, "Approximation of failure probability using conditional sampling," $12^{\text {th }}$ AIAA/ISSMO Multidisciplinary Analysis and Optimization Conference, Sep. 2008.

[3] M. W. Long and J. D. Narciso, Report DOT/FAA/AR99/2 - Probabilistic Design Methodology for Composite Aircraft Structures (unpublished), 1999.

[4] C. Hindman, Document \#TR022931 - Thrust Vector Misalignment Model Report (unpublished), ATK Launch Systems, Brigham City, UT, 2008.

[5] NASA, "Rocket Motor Test Helps NASA's Shuttle and Ares I," Dec. 4, 2008. [Online]. Available: http://www.nasa.gov/centers/marshall/multimedia/photo s/2008/photos08-176.html. [Accessed: Apr. 3, 2008].

[6] P. A. Parker, Ares I Static Test Parameter Estimation (unpublished), Langley Research Center, Hampton, VA, 2008. 